Spoken sessions

Abstract S47 Table 1. Median treatment effects on PVR and CI

\begin{tabular}{|c|c|c|c|c|c|c|c|c|}
\hline & \multicolumn{4}{|c|}{$\begin{array}{l}\text { PVR, dyn.sec/cm } \\
\text { (relative benefit* to placebo expressed in \%) }\end{array}$} & \multicolumn{4}{|l|}{$\mathrm{Cl}, \mathrm{L} / \mathrm{min} / \mathrm{m}^{2}$} \\
\hline & Macitentan 3mg & $p$-value & Macitentan $10 \mathrm{mg}$ & $p$-value & Macitentan 3mg & $p$-value & Macitentan $10 \mathrm{mg}$ & $\mathrm{p}$-value \\
\hline All & $-28.7(-32.2,-19.2)$ & $<0.0001$ & $-37.4(-46.3,-26.6)$ & $<0.0001$ & $0.5(0.3,0.8)$ & $<0.0001$ & $0.6(0.4,0.9)$ & $<0.0001$ \\
\hline Treatment Naïve & $-19.9(-34.2,0.8)$ & 0.06 & $-40.3(-52.0,-22.3)$ & 0.0002 & $0.4(0.1,0.8)$ & 0.01 & $0.6(0.2,1.0)$ & 0.004 \\
\hline Treated & $-34.4(-45.6,-22.3)$ & $<0.0001$ & $-33.3(-45.6,-20.7)$ & 0.0001 & $0.6(0.4,1.0)$ & $<0.0001$ & $0.6(0.2,1.0)$ & 0.005 \\
\hline $\mathrm{FC} \mathrm{I} / \mathrm{II}$ & $-35.2(-49.2,-21.6)$ & $<0.0001$ & $-46.0(-57.2,-28.8)$ & $<0.0001$ & $0.5(0.2,0.9)$ & 0.002 & $0.7(0.2,1.1)$ & 0.005 \\
\hline FC III/IV & $-21.8(-37.8,-9.2)$ & 0.002 & $-29.0(-43.8,-16.6)$ & 0.0003 & $0.5(0.3,0.9)$ & 0.0001 & $0.6(0.3,1.0)$ & 0.001 \\
\hline
\end{tabular}

Median $(95 \% \mathrm{CL}$ ) placebo-corrected change from baseline and Wilcoxon test $\mathrm{p}$-values; *based on the log of Month 6/baseline values

only chance of cure. Data on the long term survival after PEA are limited.

Method All patients who have undergone a PEA for CTEPH at Papworth hospital were included between January 1997 and November 2012. Patients who had a re-do operation were excluded. Pre- and post-operative data on haemodynamics, exercise capacity, functional class and targeted PAH therapies taken were obtained from our $\mathrm{PH}$ database and from other UK PH centres. The long-term survival of patients who returned for follow-up at 3 months post PEA was determined using the NHS spine summary care record tracking system. Overseas patients were censored when last seen.

Results 880 patients underwent PEA over the 15 year period. The mean age was 57 (range 15-84) and 53\% were male. The majority $(89 \%)$ were in WHO functional class 3 or 4 prior to surgery with an average mean pulmonary artery pressure (mPAP) of $47 \mathrm{mmHg}$ and PVR of 795 dynes. $65 \%$ of patients were taking at least 1 targeted therapy as a "bridge to surgery". Post surgery the majority of patients $(86 \%)$ were in WHO functional class 1 or 2 at the 12 month follow-up with only $17 \%$ taking targeted therapy. There was a reduction in the average mPAP to $27 \mathrm{mmHg}$ and PVR to 308 dynes by 12 months. The 10 year conditional survival post PEA of the first 314 patients from the cohort (Freed et al. J Thorac Cardiovasc Surg, 2011;141:383-7) was 74\%.

Conclusion The conditional survival of a subset of the cohort at 10 years was $74 \%$. There was a significant functional and haemodynamic improvement in the majority of patients at 12 months post surgery. Only $17 \%$ of patients at 12 months post surgery were being treated with targeted therapy.

Acknowledgements The authors would like to acknowledge the pulmonary hypertension centres in the UK. "This research was supported by the National Institute for Health Research (NIHR) Cambridge Biomedical Research Centre".

\section{S47 EFFECT OF MACITENTAN ON HAEMODYNAMICS IN PATIENTS WITH PULMONARY ARTERIAL HYPERTENSION: RESULTS FROM THE LONG-TERM, RANDOMISED, PLACEBO-CONTROLLED SERAPHIN TRIAL}

${ }^{1} \mathrm{G}$ Coghlan, ${ }^{2} \mathrm{~A}$ Torbicki, ${ }^{3} \mathrm{~N}$ Galiè, ${ }^{4} \mathrm{U}$ Rubin, ${ }^{5} \mathrm{~L}$ Perchenet, ${ }^{6} \mathrm{G}$ Simonneau; ${ }^{1}$ Royal Free Hospital, London, United Kingdom; ${ }^{2}$ Department of Pulmonary Circulation and Thromboembolic Diseases, Center of Postgraduate Medical Education, ECZ-Otwock, Poland; ${ }^{3}$ University of Bologna, Bologna, Italy; ${ }^{4}$ Division of Pulmonary \& Critical Care Medicine, University of California, San Diego, USA; ${ }^{5}$ Actelion Pharmaceuticals Ltd, Allschwil, Switzerland; ${ }^{6}$ Service de Pneumologie, Hôpital Universitaire de Bic\tre, Le Kremlin Bic \tre, France

\subsection{6/thoraxjnl-2013-204457.54}

Introduction and objectives Macitentan, a novel dual endothelin receptor antagonist (ERA), significantly reduced morbidity and mortality in pulmonary arterial hypertension (PAH) patients in the SERAPHIN trial (NCT00660179), the first event-driven outcomes trial in PAH. A substudy in SERAPHIN investigated the effect of macitentan on patients' cardiac haemodynamics.

Methods $742 \mathrm{PAH}$ patients were randomised to placebo, macitentan $3 \mathrm{mg}$, or macitentan $10 \mathrm{mg}$ once-daily. Stable background $\mathrm{PAH}$ therapy, except injectable prostanoids and other ERAs, were allowed. At selected centres, patients underwent right heart catheterisation at randomisation and Month 6 . Changes from baseline to Month 6 for mean right atrial pressure (mRAP), mean pulmonary arterial pressure (mPAP), pulmonary vascular resistance (PVR), cardiac index (CI) and mixed venous oxygen saturation $\left(\mathrm{SvO}_{2}\right)$ were calculated for all patients and stratified in an exploratory analysis for background PAH therapy and baseline WHO functional class I/II vs III/IV. Median treatment effects (95\% CL) between placebo and macitentan are reported.

Results 187 patients participated in the substudy (51\% were treatment-naïve and 56\% in WHO FC III/IV). Baseline median values for all patients on placebo $(\mathrm{n}=68)$, macitentan $3 \mathrm{mg}$ $(\mathrm{n}=62)$ and $10 \mathrm{mg}(\mathrm{n}=57)$ were: $\mathrm{mRAP} 7.0,8.0,7.0 \mathrm{mmHg}$; mPAP 52.0, 54.0, $52.3 \mathrm{mmHg}$; PVR 800, 785, $789 \mathrm{dyn} \cdot \mathrm{sec} / \mathrm{cm}^{5}$; CI 2.49, 2.23, $2.47 \mathrm{~L} / \mathrm{min} / \mathrm{m}^{2}$; and $\mathrm{SvO}_{2}$ 66.0, 64.5, 66.5\%, respectively. Overall, haemodynamic parameters improved at Month 6 with macitentan and worsened with placebo. Beneficial treatment effects with macitentan were statistically significant (P $<0.05$ ) for PVR and CI for both subgroups, except for PVR in treatment naïve patients treated with macitentan $3 \mathrm{mg}$ (Table).

Conclusions Macitentan significantly improved cardio-pulmonary haemodynamics in PAH patients. Improvements in PVR and $\mathrm{CI}$ were consistent irrespective of background PAH therapy and baseline WHO FC.

\section{S48 INEFFICIENT VENTRICULO-ARTERIAL COUPLING CONTRIBUTES TO REDUCED EXERCISE CAPACITY IN PULMONARY HYPERTENSION}

C McCabe, Hoole, P White, R Axell, L Shapiro, J Pepke-Zaba; Papworth Hospital, Cambridge, United Kingdom

\subsection{6/thoraxjnl-2013-204457.55}

Introduction Ventriculo-arterial (VA) coupling (Ees/Ea) in the right heart is defined by RV end-systolic elastance (Ees) and pulmonary arterial effective elastance $(\mathrm{Ea})$ with Ees/Ea representing the mechanical efficiency of forward flow from the RV. Ees/Ea may influence exercise capacity in pulmonary hypertension $(\mathrm{PH})$ because patients exhibit cardiac limitation at peak oxygen uptake (peak $\mathrm{VO}_{2}$ ) and suffer impaired exercise cardiac output adaptation. We hypothesised that Ees/Ea in the RV represents a 
physiological index of myocardial reserve and thus at inefficient ratios, may predispose to reduced exercise capacity.

Methods Using RV conductance catheterisation and contemporaneous incremental cardiopulmonary exercise testing, we evaluated Ees/Ea against peak $\mathrm{VO}_{2}$ in twenty patients with pulmonary vascular disease. Ees/Ea was compared with haemodynamic predictors of exercise capacity obtained from standard right heart catheterisation.

Results Resting Ees/Ea, absolute peak $\mathrm{VO}_{2}$ and predicted peak $\mathrm{VO}_{2}$ were $0.86 \pm 0.40,19.6 \pm 6.7 \mathrm{ml} / \mathrm{Kg} / \mathrm{min}$ and $88 \pm 23 \%$ respectively. Univariable predictors of absolute peak $\mathrm{VO}_{2}$ were patient gender, NYHA class, mean right atrial pressure, mean pulmonary artery pressure, cardiac index, conductance RV stroke volume and Ees/Ea (all $\mathrm{p}<0.10$ ). On bivariate analysis, the predictive value of Ees/Ea improved following adjustment for RV stroke volume $(\mathrm{p}=0.03)$ but not for mean RA pressure $(\mathrm{p}=0.21)$. Only Ees/Ea related linearly to percent predicted $\mathrm{VO}_{2}\left(\mathrm{R}^{2}=0.32, \mathrm{p}=0.01\right) . \mathrm{RV}$ diastolic decay $\left(-\mathrm{dP} / \mathrm{dt}_{\min }\right)$ showed good correlation with $\mathrm{O}_{2}$ pulse evolution $(\mathrm{r}=0.62, \mathrm{p}$ $<0.01$ ) although no single haemodynamic parameter differentiated absolute peak $\mathrm{VO}_{2}$ above and below its median value.

Discussion VA coupling is a marker of RV energetic efficiency and adds to the debate on the multifactorial determinants of exercise capacity in $\mathrm{PH}$. Ees/Ea was comparable to other predictive haemodynamic parameters of exercise capacity and may represent the 'recruitable' myocardial reserve, important for maintaining cardiac output at increased metabolic demand. Ees/Ea may be a potential therapeutic target given the unclear relationship between pulmonary haemodynamics and patient symptoms.

\begin{tabular}{|c|c|c|c|c|}
\hline & \multicolumn{2}{|c|}{ Univariable analysis } & \multicolumn{2}{|c|}{ Univariable analysis } \\
\hline & \multicolumn{2}{|c|}{ Peak $\mathrm{VO}_{2} \mathrm{ml} / \mathrm{Kg} / \mathrm{min}$} & \multicolumn{2}{|c|}{ Peak $\mathrm{VO}_{2} \%$ predicted } \\
\hline & $\beta$ & P Value & $\beta$ & P Value \\
\hline \multicolumn{5}{|l|}{ Clinical } \\
\hline Age & -0.16 & 0.50 & 0.22 & 0.34 \\
\hline Gender & 0.53 & 0.02 & -0.25 & 0.29 \\
\hline BSA & -0.33 & 0.16 & -0.25 & 0.30 \\
\hline NYHA Class & -0.62 & $<0.01$ & -0.18 & 0.44 \\
\hline \multicolumn{5}{|l|}{ Swan Ganz } \\
\hline Mean RAP $(\mathrm{mmHg})$ & -0.42 & 0.06 & -0.30 & 0.21 \\
\hline mPAP $(\mathrm{mmHg})$ & -0.56 & 0.01 & -0.38 & 0.10 \\
\hline Cardiac Index (L/min/m2) & 0.60 & $<0.01$ & 0.21 & 0.38 \\
\hline \multicolumn{5}{|l|}{ Conductance } \\
\hline RVSW & -0.12 & 0.61 & -0.37 & 0.11 \\
\hline sv & 0.39 & 0.09 & -0.08 & 0.74 \\
\hline Ees $(\mathrm{mmHg} / \mathrm{ml})$ & 0.06 & 0.79 & -0.18 & 0.45 \\
\hline Ea $(\mathrm{mmHg} / \mathrm{ml})$ & -0.29 & 0.22 & -0.34 & 0.14 \\
\hline $\mathrm{Ca}(\mathrm{ml} / \mathrm{mmHg})$ & 0.33 & 0.16 & 0.14 & 0.54 \\
\hline Ees/Ea & 0.45 & 0.04 & 0.56 & 0.01 \\
\hline
\end{tabular}

\section{S49 THE DIAGNOSTIC VALUE OF MEASURING AAG DURING EXERCISE IN PATIENTS WITH PULMONARY HYPERTENSION}

B Mukherjee, E Chan, K Murphy, H Tighe, R Davies, S Gibbs, L Howard; Hammersmith Hospital, Imperial College, London, UK

10.1136/thoraxjnl-2013-204457.56
The exercise response in pulmonary hypertension $(\mathrm{PH})$ has characteristic features, including decreased peak oxygen consumption (VO2-peak), increased ventilatory inefficiency (VE/ $\mathrm{VCO} 2$ slope) and widened alveolar-arterial oxygen-gradient (AaG). We wished to evaluate if the AaG at peak exercise predicted those patients likely to have $\mathrm{PH}$ who would subsequently require catheter studies.

Methods We performed a retrospective analysis of patients referred to Hammersmith Hospital between Feb 2008 and Feb 2012 for investigation of Pulmonary Hypertension $(\mathrm{PH})$ who underwent cardiopulmonary exercise testing (CPX) with testing of AaG using arterial blood gas analysis at peak exercise. Patients found to have alternative cardiac or respiratory diagnoses were excluded. Patients given diagnoses of Pulmonary Arterial Hypertension or Pulmonary Hypertension due to Left Heart Disease and with temporally coincident data from CPX and RHC (within 3 months) were included. Patients without cardiorespiratory diagnoses were healthy controls. The VE/VCO2 slope and AaG were compared to the diagnosis of $\mathrm{PH}$ and the trans-pulmonary pressure gradient (TPG), (the difference between mean pulmonary artery pressure (mPAP) and pulmonary capillary wedge pressure (PCWP) or left ventricular end diastolic pressure (LVEDP) where available).

Results Using logistic regression to predict a diagnosis of $\mathrm{PH}$, AaG had an odds ratios of 2.98 ( $\mathrm{p}<0.01)$ and receiver operating characteristic curve for sensitivity and specificity had area under the curve (ROC-AUC) of 0.92. An AaG cut-off of $2.5 \mathrm{kPA}$ had $90 \%$ sensitivity and $80 \%$ specificity. Similarly, VE/VCO2 had an odds ratio of $1.21(\mathrm{p}<0.01)$ and ROC-AUC 0.85 for predicting $\mathrm{PH}$. Combining AaG and VE/VCO2 had ROC-AUC of 0.94 for diagnosing $\mathrm{PH}$ without significant interaction between $\mathrm{AaG}$ and VE/VCO2. For predicting a TPG $>12 \mathrm{mmHg}$, $\mathrm{AaG}$ had an odds ratios of $4.54(\mathrm{p}<0.01)$ and ROC-AUC of 0.95 . VE/VCO2 had an odds ratio of $1.10(\mathrm{p}<0.01)$ and ROCAUC 0.74 for predicting TPG $>12 \mathrm{mmHg}$.

Conclusion CPX has become part of the diagnostic workup of patients with $\mathrm{PH}$. AaG measured at peak exercise has a high sensitivity and specificity in predicting patients with $\mathrm{PH}$, which may help determining which patients will require invasive catheter studies. The AaG provides independent information than VE/VCO2 alone in predicting $\mathrm{PH}$ and may be useful in the investigation of $\mathrm{PH}$.

\section{S50 HYPERSENSITIVITY PNEUMONITIS COMPLICATED BY PULMONARY HYPERTENSION; PATIENT CHARACTERISTICS AND RESPONSE TO TARGETED THERAPY}

BE Garfield, GJ Keir, LC Price, AU Wells, E Renzoni, TM Maher, P Marino, K Dimopoulos, SJ Wort; Royal Brompton and Harefield NHS Foundation Trust, London, UK;

\subsection{6/thoraxjnl-2013-204457.57}

Background Hypersensitivity pneumonitis (HP) results from repeated exposure to a sensitizing antigen, normally an organic particle. It can be acute, sub-acute or chronic (1). There is very little literature describing the association of pulmonary hypertension $(\mathrm{PH})$ with HP (2). We aimed to summarize the clinical characteristics and outcomes including responses to targeted therapy in patients with $\mathrm{HP}$ and $\mathrm{PH}$ in a tertiary referral centre.

Methods Cases diagnosed between 1992 and 2008 were identified through a central database. $\mathrm{PH}$ was defined as mean pulmonary artery pressure $\geq 25 \mathrm{mmHg}$ on right heart catheter or right ventricular systolic pressure of $\geq 50 \mathrm{mmHg}$ on echocardiogram. Data was collected through case note and electronic record review. Analysis was performed using Graphpad prism. 